\title{
Identification of the complex relative dielectric constant of porous polymers at different degrees of humidity
}

\author{
K. Haake and J. L. ter Haseborg \\ Hamburg University of Technology, Institute for Metrology and EMC, Germany
}

\begin{abstract}
In order to investigate the complex relative dielectric constant of different materials over a frequency range of $50 \mathrm{MHz}$ to $3 \mathrm{GHz}$ a setup has been built that allows rfmeasurements in a climate chamber. Within this chamber the temperature and humidity can be changed and be measured by accurate sensors. The construction of the rf-part of the setup has been made with an outer cylindrical shell that allows climate interactions through its large orifices. Methods for calculating the complex relative dielectric constant from the measured scattering parameters are presented and the changes of the relative constants due to different humidity conditions are shown.
\end{abstract}

\section{Introduction}

Constructions in rf-metrology often use wood as nonconducting material. Wood combines the advantages of easy workability, low weight, high mechanical stability, low cost and a low relative permittivity compared to a lot of other nonconducting materials. The last point results on the fact that wood is a more or less porous material. This is the reason for the assumed disadvantage of wood, making it acting like a sponge for humidity. This might alter its electric behavior causing different diffraction and especially raises the dielectric losses of the material. To know the influence of the different materials under different environmental conditions is highly interesting for rf-metrology. Hence the investigation of this effect and especially the influence of humidity towards the dielectric behavior is the goal of this paper. Due to the fact that the relative dielectric constant is strongly dependent on frequency, a setup has been built that permits measurements up to $3 \mathrm{GHz}$. Further the humidity of the environment

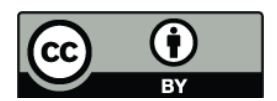

Correspondence to: K. Haake (k.haake@tu-harburg.de) can be altered. The result of the measurement is a determination of the complex relative dielectric constant:

$\underline{\varepsilon_{r}}(f)=\varepsilon_{r}^{\prime}(f)-j \cdot \varepsilon_{r}^{\prime \prime}(f)$

\section{Measurement setup}

The setup, depicted in Fig. 1, consists of a coaxial transmission line (Unger, 1995) that is shown in Fig. 2. It is tapered at both sides in order to enlarge the diameter in the middle segment without drastically change the wave impedance, keeping it in the vicinity of $50 \Omega$. The dielectric in this section is the specimen to study. Due to the fact that the influence of humidity towards the dielectric is to be investigated, the middle section's cylindrical shell is been simulated by using wires symmetrically positioned around the circumference as shown in Fig. 1. Such an outer wire conductor keeps up the coaxial structure and electrical behavior of the transmission line as shown by Rojas-Coto and ter Haseborg (2006) and further permits an interchange of the environmental climate. The coaxial setup is placed inside a sealed box, which is only been penetrated by cables and a small tube for the water supply. Inside are also an evaporator and a fan (with heating) for homogenizing the climate conditions within the box. Two identical sensors are placed inside the box, which are able to measure the relative humidity, temperature and dew point. Further parts of the setup are a vector network analyzer and an interface to digitalize the temperature and humidity signals digitally for further processing. A PC controls the data acquisition and stores the measurements.

\section{Theory}

The complex relative dielectric constant will be calculated using the scattering parameters (Zamow, 2005). These have been measured beforehand by the vector network analyzer 


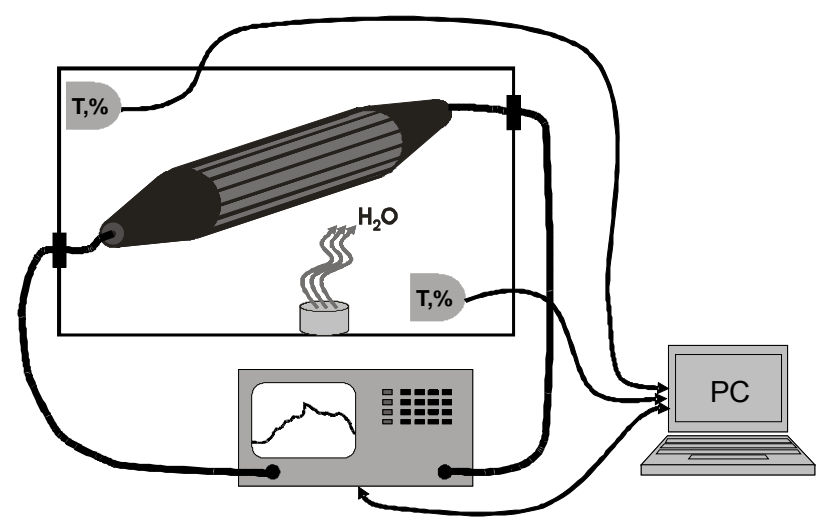

Fig. 1. Measurement setup with climate chamber.

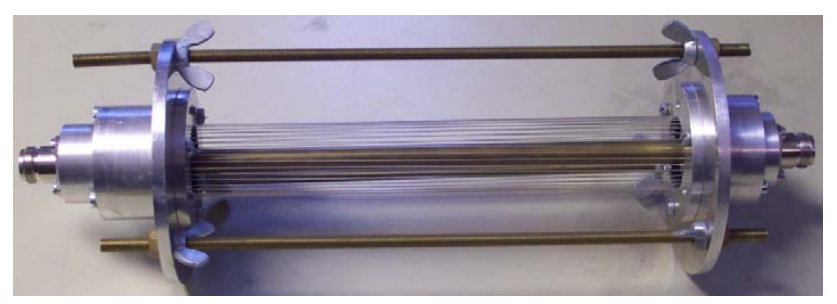

Fig. 2. Coaxial setup with outer weir conductor in the middle section.

and are put into the $2 \times 2$-scattering-matrix $\mathbf{T}$. The coaxial setup consists of different sections $(A-B-C-X-D-E-F)$ with different wave impedances, but only the middle part $X$ is filled with the specimen. Measuring the coaxial setup with a TDR, it can be divided into sections as depicted in Fig. 3.

Section $X$ denotes the specimen section. Hence $\mathbf{T}$ has to be transformed to chain matrix notation and Eq. (2) can be applied as shown in Schiek (1984).

$\mathbf{T}=\mathbf{A} \cdot \mathbf{B} \cdot \mathbf{C} \cdot \mathbf{X} \cdot \mathbf{D} \cdot \mathbf{E} \cdot \mathbf{F}$

In order to calculate the middle section $X$, Eq. (3) is used and a back transformation to scattering parameters has to be done afterwards.

$\mathbf{X}=\mathbf{C}^{-1} \cdot \mathbf{B}^{-1} \cdot \mathbf{A}^{-1} \cdot \mathbf{T} \cdot \mathbf{F}^{-1} \cdot \mathbf{E}^{-1} \cdot \mathbf{D}^{-1}$

This is necessary, because the Nicholson-Ross method (Eq. 4) as used by Zamow (2005) uses the scattering parameters of $\mathrm{X}$ to calculate the complex relative dielectric constant of this section.

$\varepsilon_{r, \mathrm{eff}}=\sqrt{\frac{-\left[\frac{c_{0}}{\omega l_{x}} \ln \left(\frac{S_{X 11}+S_{X 21}-R_{12}}{1-\left(S_{X 11}+S_{X 21}\right) R_{12}}\right)\right]}{\left(\frac{1+R_{12}}{1-R_{12}}\right)^{2}}}$

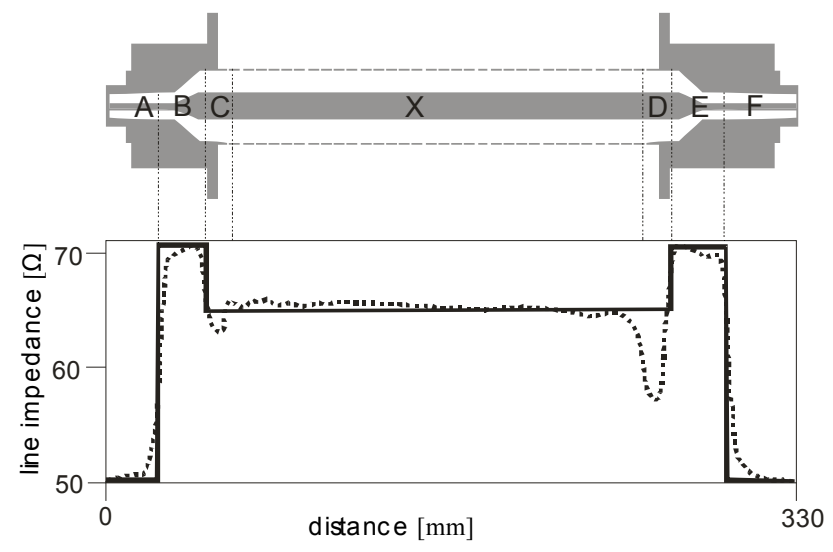

Fig. 3. Coaxial measurement section (top) with TDR measurement. TDR plot has been stretched piecewise making the $\mathrm{x}$-axis linearly scalable.

The terms for $W$ and $R_{12}$ are given in Eqs. (5) and (6); the absolute value of Eq. (5) is supposed to be equal or smaller than 1 in order to get physically sensible results.

$W=\frac{1-\left(S_{X 21}^{2}-S_{X 11}^{2}\right)}{2 S_{X 11}} \pm \sqrt{\left(\frac{1-\left(S_{X 21}^{2}-S_{X 11}^{2}\right)}{2 S_{X 11}}\right)^{2}-1}$

$R_{12}(\omega)=W \pm \sqrt{W^{2}-1}$

The Nicholson-Ross method tends to provide not very smooth curves (Zamow, 2005). To achieve this, the Debye function (Eq. 7) is applied. This function is supposed to express the general run of the relative dielectric constant of a material over the frequency.

$$
\begin{aligned}
\varepsilon_{r}(\omega) & =\varepsilon_{r}^{\prime}-j \varepsilon_{r}^{\prime \prime} \\
& =\left(\varepsilon_{\infty}+\frac{\varepsilon_{s}-\varepsilon_{\infty}}{1+\omega^{2} \tau_{e}^{2}}\right)-j\left(\frac{\sigma_{e}}{\omega \varepsilon_{0}}+\frac{\left(\varepsilon_{s}-\varepsilon_{\infty}\right) \omega \tau_{e}}{1+\omega^{2} \tau_{e}^{2}}\right)
\end{aligned}
$$

The parameters to determine in this function approximating the resulting run of the curve gotten by the Nicholson-Ross method are: Static permittivity $\varepsilon_{s}$, specific conductivity $\sigma_{e}$ and relaxation time $\tau_{e}$.

The relative permittivity at very high frequencies $\varepsilon_{\infty}$ approximates 1 due to the fact that at very high frequencies no polarization is possible (Zamow, 2005).

\subsection{Humidity}

The relative humidity is defined as the percentage of the absolute mass of water that can be solved in a gas at a certain temperature without condensing (Hilbrunner, 2005). This depends of course on the used gas. Hence the absolute humidity is the mass of water that is solved in a gas. Figure 4 depicts the absolute humidity for air over the temperature. The different curves show different saturation degrees. Nevertheless in Central Europe the absolute humidity hardly exceeds $20 \mathrm{~g} / \mathrm{m}^{3}$. 


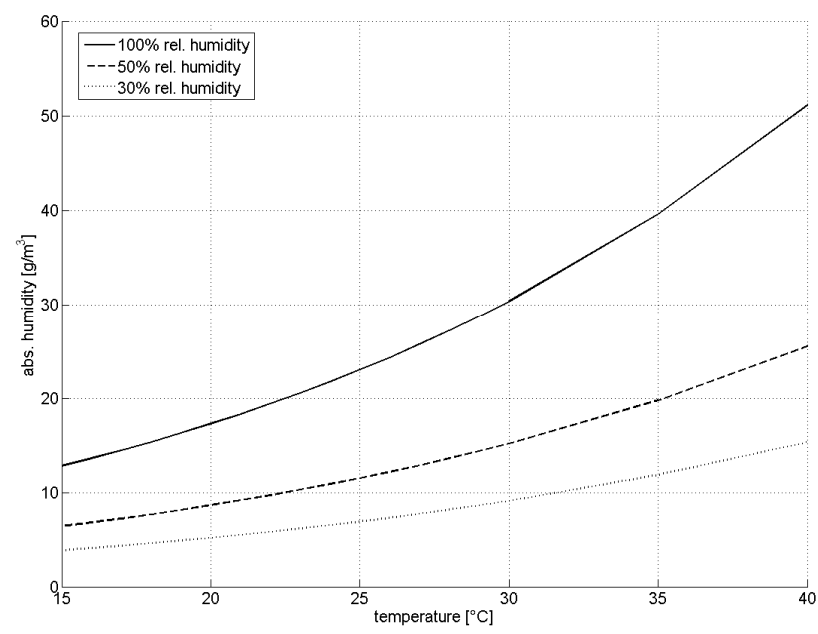

Fig. 4. Absolute humidity of air for three different saturation conditions (Hilbrunner, 2005).

\section{Results}

Two different materials have been tested that are mostly used in rf-constructions in the institute. These are balsa and beech. The electrical characteristics of different types of wood are described in Vidémé Bossou (2007). This investigation deals only with the relative change of the electrical characteristics. The results have been obtained by measuring the scattering parameters at a steady state of the wood's humidity. This has been accomplished by recording over a long time simultaneously the data of the outer environmental humidity and the scattering parameters. When the change goes to zero the data has been taken.

Figures 5 and 6 show the relative change of the complex relative dielectric constant of balsa and beech over frequency. Normalization has been done to the lowest value of measured humidity. For reasons of depiction, the real and the negative imaginary part are plotted separately. It can be observed, that despite of drastic change in humidity, the change in the relative dielectric constant is not significantly. Due to the use of Eq. (7) the resulting curves are smoother than the actual measured data.

\section{Conclusion}

A setup has been built that allows measuring the change of the complex relative dielectric constant over a frequency range of $50 \mathrm{MHz}$ to $3 \mathrm{GHz}$. The measurements have been taken out under different humidity conditions. The specialty of the setup is that the construction of the quasi coaxial conductor lets the climate have a direct effect on the specimen while still allowing measurements up to high frequencies. The results show that the effect of humidity towards the dielectric behavior and the loss of the materials is rather small.
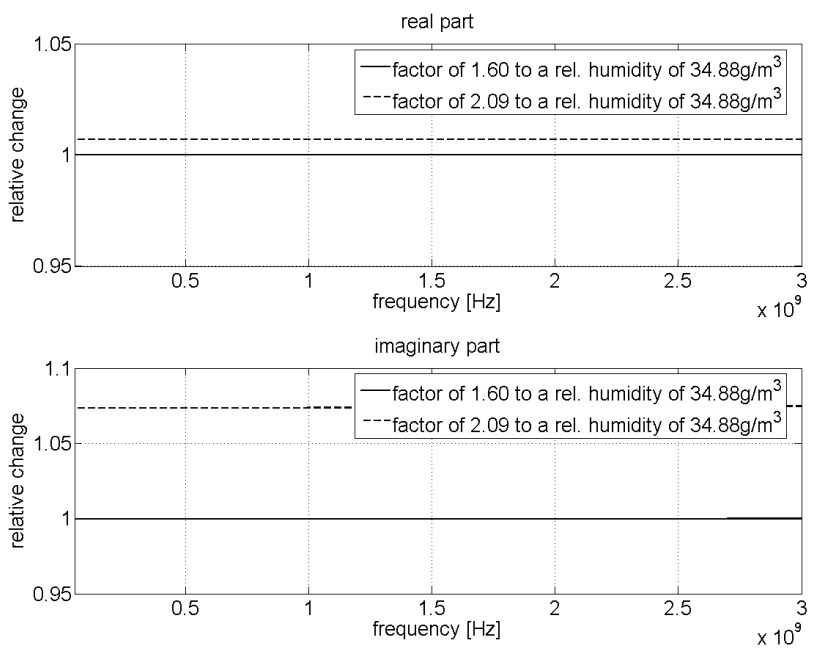

Fig. 5. The relative change of the complex relative dielectric constant of balsa shown as real part (top) and negative imaginary part (botton).
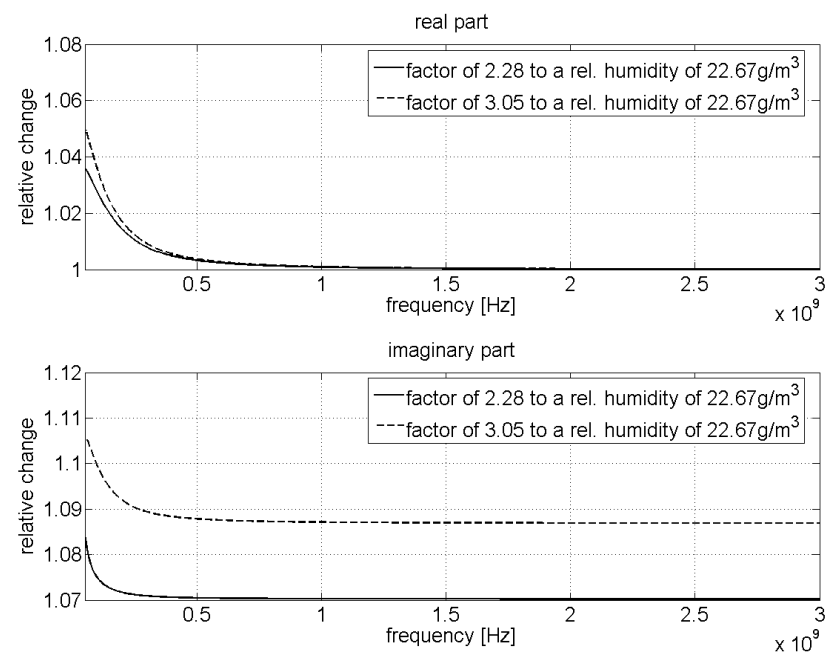

Fig. 6. The relative change of the complex relative dielectric constant of beech shown as real part (top) and negative imaginary part (botton).

\section{References}

Hilbrunner, F.: Ein Beitrag zur Feuchtekompensation von Präzisionsmeßgeräten, $\mathrm{PhD}$. Thesis, Technische Universität Ilmenau, Ilmenau, 2005.

Rojas-Coto, A. and ter Haseborg, J. L.: Bestimmung der Induktivität komplexer großer metallischer Strukturen mittels Reusenleiteraufbau für die EMV-Analyse, Int. Fachmesse und Kongress für EMV, VDE-Verlag GmbH, Offenbach, 2006.

Schiek, B.: Meßsysteme der Hochfrequenztechnik, Hüthig, Heidelberg, 1984.

Unger, H.-G.: Elektromagnetische Wellen auf Leitungen Hüthig, Heidelberg, 1995. 
K. Haake and J. L. ter Haseborg: Identification of relative dielectric constant of porous polymers

Vidémé Bossou, O.: A simple \& accessible front-end satellite receiver for communication and e-learning, PhD. Thesis, Ecole Polytechnique Fédérale de Lausanne, Lausanne, 2007.
Zamow, D.: Untersuchung und Simulation des Absorptionsverhaltens in TEM-Wellenleitern, diploma thesis, Universität Hannover, 2006. 